\title{
Thermal Radiation Effect on Unsteady Convection and Mass Transport Over a Stretching Sheet in a Saturated Porous Medium With Chemical Reaction
}

Md. Hasanuzzaman ( $\sim$ hasanuzzaman@math.kuet.ac.bd)

Khulna University of Engineering and Technology

Romana Afroj

Khulna University of Engineering and Technology

Shanta Sharin

Khulna University of Engineering and Technology

Akio Miyara

Saga University

Research Article

Keywords: Stretching surface, heat and mass transfer, thermal radiation, porous medium, chemical reaction

Posted Date: March 1st, 2022

DOI: https://doi.org/10.21203/rs.3.rs-1254356/v1

License: (9) This work is licensed under a Creative Commons Attribution 4.0 International License.

Read Full License 


\title{
Thermal Radiation Effect on Unsteady Convection and Mass Transport over a Stretching Sheet in a Saturated Porous Medium with Chemical Reaction
}

\author{
${ }^{1}$ Md. Hasanuzzaman*, ${ }^{1}$ Romana Afroj, ${ }^{1}$ Shanta Sharin and ${ }^{2,3}$ Akio Miyara \\ ${ }^{1}$ Department of Mathematics, Khulna University of Engineering \& Technology, Khulna- \\ 9203, Bangladesh \\ ${ }^{2}$ Department of Mechanical Engineering, Saga University, Saga-shi, 840-8502, Japan \\ ${ }^{3}$ International Institute for Carbon-Neutral Energy Research, Kyushu University, \\ Fukuoka-shi, 819-0395, Japan \\ Corresponding author: hasanuzzaman@math.kuet.ac.bd
}

\begin{abstract}
The impact of chemical reaction on unsteady convection and mass transport over a stretching sheet in a saturated permeable medium with the thermal radiation effect has been examined numerically. The governing partial differential equations (PDEs) have been converted into ordinary differential equations (ODEs) by using the local similarity transformation. The converted governing ODEs have been then solved numerically using the shooting technique in 'ODE45 MATLAB' software. The influence of emerging dimensionless numbers/parameters such as the thermal radiation parameter $(R)$, Prandtl number $(P r)$, Schmidt number $(S c)$, Darcy number $(D a)$ and chemical reaction parameter $\left(K_{r}\right)$ etc. on the flow, concentration and temperature distributions are presented and discussed. The fluid velocity decreases for uplifting values of the Darcy number. The fluid temperature enhances for rising values of the thermal radiation parameter. The concentration reduces for rising values of the chemical reaction parameter. The local skin-friction coefficient decreases about $21 \%$ due to increasing the Darcy number (0.6-1.6). The heat transfer rate decreases about $25 \%$, and increases about $22 \%$ due to increasing the thermal radiation parameter (0.5-1.0) and the Prandtl number (0.71-1.0), respectively. The mass transfer rate increases about $9 \%$, and $46 \%$, due to increasing the chemical reaction parameter (0.5-1.0), and the Schmidt number (0.5-1.0), respectively. Finally, we compared our numerical results with previously published literature and observed to be a good agreement.
\end{abstract}

Keywords: Stretching surface, heat and mass transfer, thermal radiation, porous medium, chemical reaction 


\section{Nomenclature}

$\begin{array}{clcl}u & \text { velocity component along the x-axis } & v & \text { velocity component along the y-axis } \\ T & \text { temperature of fluid } & C & \text { fluid concentration } \\ T_{S} & \text { surface temperature } & C_{S} & \text { surface concentration } \\ T_{r e f} & \text { reference temperature } & C_{r e f} & \text { reference concentration } \\ T_{0} & \text { temperature at the slit } & C_{0} & \text { concentration at the slit } \\ U(x, t) & \text { uniform surface velocity } & h(t) & \text { thin liquid film thickness } \\ \alpha & \text { thermal diffusivity } & \rho & \text { fluid density } \\ \mu & \text { dynamic viscosity } & v & \text { kinematic viscosity } \\ K & \text { permeability of surface } & D^{*} & \text { coefficient of mass diffusion } \\ C_{p} & \text { specific heat at constant pressure } & K^{\prime} & \text { chemical rate } \\ \eta & \text { similarity variable } & f_{\eta}(\eta) & \text { dimensionless velocity } \\ \theta(\eta) & \text { dimensionless temperature } & \phi(\eta) & \text { dimensionless concentration } \\ S_{c} & \text { Schmidt number } & P_{r} & \text { Prandtl number } \\ k & \text { thermal conductivity } & q_{r} & \text { radiative heat flux } \\ t & \text { time } & \sigma^{*} & \text { Stefan-Boltzmann constant } \\ K^{*} & \text { mean absorption coefficient } & R & \text { radiation parameter } \\ D a & \text { Darcy number } & K_{r} & \text { chemical reaction } \\ C_{f} & \text { local skin-friction coefficient } & N u_{x} & \text { local Nusselt number } \\ S h_{x} & \text { local Sherwood number } & R e_{x} & \text { local Reynolds number } \\ f_{\eta \eta}(0) & \text { local skin friction coefficient } & \theta_{\eta}(0) & \text { heat transfer rate } \\ \phi_{\eta}(0) & \text { mass transfer rate } & & \\ & & & \\ & & & \end{array}$

\section{Introduction}

In last two decades many problems in a liquid thin film flows on stretching medium have been widely analyzed due to their enormous applications. The study of the flow field in a boundarylayer near a stretching plate is a momentous portion in heat transfer and fluid dynamics happening in many engineering processes for example rubber sheets and extrusion of plastic, metallurgy and processing of polymer. When the excluded material is marked by a die in the melt spinning procedure then the flow involved because of stretching media is very near the excluded material is an example. The characteristic of heat transfer and flow in a liquid thin film allow us to get the desired ending of the coating even in the development and design of 
several chemical processing equipment's and heat exchangers. The study of flow features in a liquid thin film reposing on an unsteady stretching plate has been initiated by Wang [1]. He used the familiar technique which is similarity transformation to convert the governing PDEs to a non-linear ODEs in the presence of an unsteadiness parameter. Dandapat et al. [2] extended this analysis to add the analysis of heat transfer. The analysis of fluid flow and heat transfer passing in a permeable media has adopted noticeable attention owing to many thermal engineering in several branches, for example pollution of ground water, processes of cooling of electronic components, geophysical thermal insulation, oil recovery, geothermal extraction, radioactive nuclear waste materials storage, welding, food processing and casting of manufacturing processes. The joint natural and forced convective flow about continuous surface in permeable medium has been investigated by Cheng [3]. The effect of constant surface temperature on dual solutions happening in the problem of the forced and free convective flow on a vertical surface in permeable media for the event of opposing flow has been studied by Merkin [4]. Wright et al. [5] investigated the impact of a prescribed surface heat flux on natural convective flow from a vertical sheet in permeable medium. The impact of uniform lateral mass flux on free convective flow about a cone embedded in a saturated permeable medium has been analyzed by Yih [6]. The impact of surface mass transfer on natural and forced convective flow in non-Newtonian fluids in permeable medium has been investigated by Gorla and Takhar [7]. The similarity solution for the two-dimensional steady boundary layer flow caused by the flat stretching plate has been analyzed by Crane [8]. Gupta and Gupta [9] examined the heat and mass transport over a stretching plate with blowing or suction effect. Later Wang's problem for the case of heat transfer has been extended by Andersson et al. [10]. Abel et al. [11] studied the impact of viscous dissipation on the unsteady heat transfer and flow in a liquid film over a stretching surface with the magnetic field effect. The impact of magnetic field on an unsteady elastic stretching plate in a thin liquid film with thermocapillary effect has been investigated by Noor and Hashim [12]. The relationships of chemical reaction and mass transfer are very tough to study. This relationship can be analyzed in the utilization of production and reactant species at several rates within the mass transfer of fluid. Chemical reaction in a flow introduced by a stretched plate is momentous in plastic extrusion, metallurgy, and biochemical engineering. A few examples involve temperature and moisture provides across an energy transmission in a rainy cooling tower or agricultural plot. A lot of researches have been discussed in connection to these applications. The impact of double diffusion on the chemically reactive stream of a hybrid nanofluid in the presence radiation has been elaborated by Song et al. [13]. The finite difference method (FDM) performs 
a significant rule in current researches in this field. It has been displayed that this method is a strong tool for solving several kinds of problems (Faisal et al. [14], Sweilam et al. [15], Cheng and Liu [16], Johnston and Liu [17], Chamkha et al. [18]). This method decreases the problem to a system of algebraic equations. Hasanuzzaman et al. [19] explained the effect of transpiration on unsteady free convective and heat transfer flow around a vertical slender body. They have also used the shooting technique for solving the ODEs with the help of 'MATLAB ODE45' software. Their simulation is almost the same as our simulation. Hasanuzzaman et al. [20] investigated the unsteady free magneto-convective heat-mass transport passing in an infinite vertical permeable sheet in the presence of Dufour and thermal diffusion effects. Khader and Megahed [21] introduced the impact of thermal radiation on an unsteady heat transfer and flow in a thin liquid film past a stretching plate in a saturated porous medium. They used the FDM to solve the resulting system of algebraic equations. Also, they considered only momentum and energy equations. But we considered here momentum, energy and concentration equations. We also applied the Quasilinearization Method (QLM) to solve the non-linear ODEs.

The main outcome of this paper is to suppose an unsteady convection and mass transport over a stretching sheet in a saturated porous medium taking into account the chemical reaction and thermal radiation effects. The mail novelty of this study is to compare our results with a published paper. Another novelty of this paper is also enhanced by assuming the chemical reaction and thermal radiation under the Quasilinearization Method (QLM) which is not explained yet. Computations have been performed for a vast range of the dimensionless numbers/parameters for example thermal radiation parameter, chemical reaction parameter, Prandtl number, Schmidt number, Darcy number, unsteadiness parameter and film thickness on temperature, concentration, and velocity profiles are discussed graphically and analyzed. Besides to the properties of the heat and mass transfer, and skin friction coefficient have been explained in the tabular formations.

\section{Governing Equation}

Let us consider the unsteady Newtonian fluid flow in a thin liquid film over a stretching surface. The elastic plate issued from a narrow slit at the origin of a Cartesian coordinate system as shown in Figure 1. The stretching surface aligned with the $x$-axis at $y=0$ moves in its own plane with temperature and concentration distributions $T_{S}(x, t)$ and $C_{S}(x, t)$ and a velocity $U(x, t)$. The uniform thickness $h(t)$ of a thin liquid film lies on the horizontal surface. 


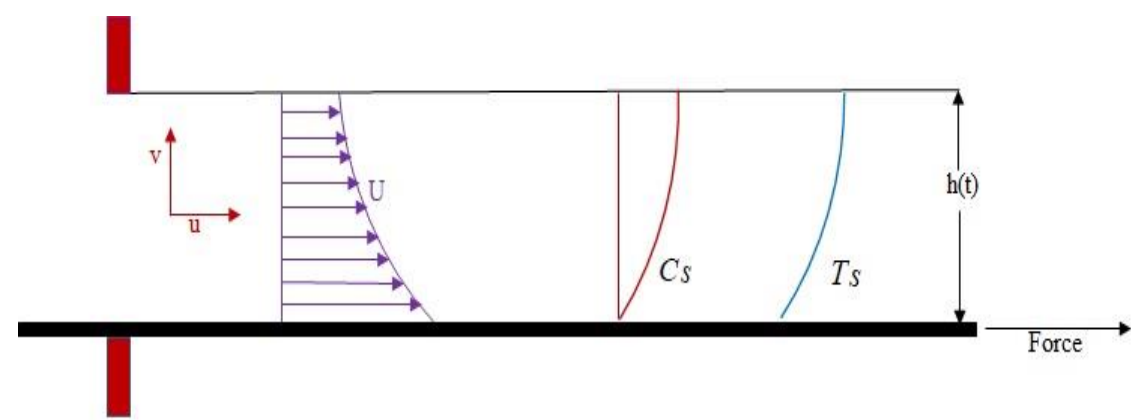

Figure 1: Physical model and coordinate system

The continuity equation, momentum equation, energy conservation equation and mass balance equation are the governing equations. The above-mentioned governing equations are given in the following forms:

The continuity equation-

$\frac{\partial u}{\partial x}+\frac{\partial v}{\partial y}=0$

The momentum equation-

$\frac{\partial u}{\partial t}+u \frac{\partial u}{\partial x}+v \frac{\partial u}{\partial y}=\frac{\mu}{\rho} \frac{\partial^{2} u}{\partial y^{2}}-\frac{\mu}{\rho K} u$

The energy conservation equation-

$\frac{\partial T}{\partial t}+u \frac{\partial T}{\partial x}+v \frac{\partial T}{\partial y}=\alpha \frac{\partial^{2} T}{\partial y^{2}}-\frac{1}{\rho c_{p}} \frac{\partial q_{r}}{\partial y}$

The mass balance equation-

$\frac{\partial C}{\partial t}+u \frac{\partial C}{\partial x}+v \frac{\partial C}{\partial y}=D^{*} \frac{\partial^{2} C}{\partial y^{2}}-K^{\prime}\left(C-C_{0}\right)$

where $u$ and $v$ are the velocity components in the $x$ and $y$ directions, respectively. $T$ is the temperature of the fluid, $C$ is the concentration of the fluid, $t$ is the time, $\mu$ is the viscosity of the fluid, $\rho$ is the density of the fluid, $K$ is the permeability of surface, $\alpha$ is the thermal diffusivity, $q_{r}$ is the radiative heat flux, at constant pressure $C_{p}$ is the specific heat, $D^{*}$ is the coefficient of mass diffusion and $K^{\prime}$ is the chemical rate.

The related boundary conditions are given by

$u=U, v=0, T=T_{S}, C=C_{S}$ at $y=0$ 
$\frac{\partial u}{\partial y}=\frac{\partial T}{\partial y}=\frac{\partial C}{\partial y}=0$ at $y=h$

$v=\frac{d h}{d t}$ at $y=h$

where $U$ is the velocity of the surface at $y=0$ and $h$ is the thickness of the liquid film. The sheet continuously moves with the velocity along $x$-direction is

$U=\frac{b x}{1-a t}$

where $a$ and $b$ are the positive constants having dimension (time) ${ }^{-1}$.

According to Rosseland approximation (Raptis [22]), the radiative heat flux $q_{r}$ is applied such that

$q_{r}=-\frac{4 \sigma^{*}}{3 K^{*}} \frac{\partial T^{4}}{\partial y}$

where the Stefan-Boltzmann constant is $\sigma^{*}$ and the mean absorption coefficient is $K^{*}$. We presume from Raptis [23] that the difference between the free stream temperature and the fluid temperature is small enough.

Expanding $T^{4}$ in a Taylor series about $T_{0}$ and ignoring the higher order terms, then we get:

$T^{4} \cong 4 T_{0}^{3} T-3 T_{0}^{4}$

The temperature of the surface is $T_{S}$ and the concentration of the surface is $C_{S}$ of the stretching plate varies with the distance $x$ and time $t$ in the form:

$$
\begin{aligned}
& T_{S}=T_{0}-T_{\text {ref }}\left(\frac{b \rho x^{2}}{2 \mu}\right)(1-a t)^{-\frac{3}{2}} \\
& C_{S}=C_{0}-C_{\text {ref }}\left(\frac{b \rho x^{2}}{2 \mu}\right)(1-a t)^{-\frac{3}{2}}
\end{aligned}
$$

where for all $t<\frac{1}{a}, C_{0}$ and $T_{0}$ are the concentration and temperature at the slit, respectively and the constant reference concentration and temperature are $C_{r e f}$ and $T_{r e f}$, respectively.

Now, applying the following similarity transformations: 


$$
\begin{aligned}
& \eta=\left(\frac{b \rho}{\mu}\right)^{\frac{1}{2}}(1-a t)^{-\frac{1}{2}} \beta^{-1} y \\
& u=b x(1-a t)^{-1} f_{\eta}(\eta) \\
& v=-\left(\frac{b \mu}{\rho}\right)^{\frac{1}{2}}(1-a t)^{-\frac{1}{2}} \beta f(\eta) \\
& T=T_{0}-T_{r e f}\left(\frac{b \rho x^{2}}{2 \mu}\right)(1-a t)^{-\frac{3}{2}} \theta(\eta) \\
& C=C_{0}-C_{r e f}\left(\frac{b \rho x^{2}}{2 \mu}\right)(1-a t)^{-\frac{3}{2}} \phi(\eta)
\end{aligned}
$$

Now, Noor and Hashim [12] introduced the dimensionless thin film thickness $\beta$ which is given by

$$
\begin{aligned}
& \beta=\left(\frac{b \rho}{\mu}\right)^{\frac{1}{2}}(1-a t)^{-\frac{1}{2}} h(t) \\
& f^{\prime \prime \prime}(\eta)+\gamma\left[f(\eta) f^{\prime}(\eta)-\frac{s}{2} \eta f^{\prime \prime}(\eta)-\left\{f^{\prime}(\eta)\right\}^{2}-(S+D a) f^{\prime}(\eta)\right]=0 \\
& \theta_{\eta \eta}(\eta)+\frac{P r}{(1+R)} \gamma\left[f(\eta) \theta_{\eta}(\eta)-\frac{3}{2} S \theta(\eta)-\frac{S}{2} \eta \theta^{\prime}(\eta)-2 f_{\eta}(\eta) \theta(\eta)\right]=0 \\
& \phi_{\eta \eta}(\eta)+\gamma S c\left[f(\eta) \phi_{\eta}(\eta)-\frac{3}{2} S \phi(\eta)-\frac{S}{2} \eta \phi_{\eta}(\eta)-2 \phi(\eta) f_{\eta}(\eta)-K_{r} \phi(\eta)\right]=0
\end{aligned}
$$

The converted boundary conditions are given by:

$$
\begin{aligned}
& f(0)=0, f_{\eta}(0)=1, \theta(0)=1, \phi(0)=1 \\
& f_{\eta \eta}(1)=0, \quad \theta_{\eta}(1)=0, \quad \phi_{\eta}(1)=0, f(1)=\frac{S}{2}
\end{aligned}
$$

where the suffixes of $f(\eta), \theta(\eta)$ and $\phi(\eta)$ denote derivative with respect to $\eta, S=\frac{a}{b}$ is the unsteadiness parameter, $\operatorname{Pr}=\frac{\mu C_{p}}{k}$ is the Prandtl number, $\gamma=\beta^{2}$ is the dimensionless film thickness, $R=\frac{16 \sigma^{*} T_{0}^{2}}{k^{*} k}$ is the radiation parameter, $D a=\frac{\mu(1-a t)}{\rho b k}$ is the Darcy number and $K_{r}=$ $K^{\prime}(1-a t)^{-1}$ is the chemical reaction. 


\section{Numerical Technique}

The fundamental governing equations of momentum, energy and concentration in Newtonian fluids are originally nonlinear coupled partial or ordinary differential equations. Usually, the analytical solution of these nonlinear ODEs with boundary conditions are almost difficult, so a numerical process must be made. By applying the Quasilinearization Method (QLM), the system of nonlinear ODEs (19) - (21) with the boundary conditions (22)-(23) has been solved numerically. The first concept behind this technique is the higher order non-linear differential equations are transferred into the first order simultaneous linear DEs. These first order linear DEs are further converted into initial value problems. In this consideration, we need to define new variables for these equations such as

$y_{1}=f(\eta), y_{2}=f^{\prime}(\eta), y_{3}=f^{\prime \prime}(\eta) \quad y_{4}=\theta(\eta), y_{5}=\theta^{\prime}(\eta), y_{6}=\phi(\eta)$ and $y_{7}=\phi^{\prime}(\eta)$

So, the higher-order differential equations (19)-(21) may be converted into seven equivalent first-order differential equations are

$$
\begin{aligned}
& y_{1}^{\prime}=y_{2}, y_{2}^{\prime}=y_{3}, y_{3}{ }^{\prime}=-\gamma\left[y_{1} y_{2}-\frac{s}{2} \eta y_{3}-y_{2}^{2}-(S+D a) y_{2}\right] \\
& y_{4}^{\prime}=y_{5}, \quad y_{5}{ }^{\prime}=-\frac{P r}{(1+R)} \gamma\left[y_{1} y_{5}-\frac{3}{2} S y_{4}-\frac{S}{2} \eta y_{5}-2 y_{2} y_{4}\right] \\
& \phi_{\eta \eta}(\eta)+\gamma S c\left[f(\eta) \phi_{\eta}(\eta)-\frac{3}{2} S \phi(\eta)-\frac{S}{2} \eta \phi_{\eta}(\eta)-2 \phi(\eta) f_{\eta}(\eta)-K_{r} \phi(\eta)\right]=0 \\
& y_{6}^{\prime}=y_{7}, \quad y_{7}^{\prime}=-\gamma S c\left[y_{1} y_{7}-\frac{3}{2} S y_{6}-\frac{S}{2} \eta y_{7}-2 y_{2} y_{6}-K_{r} y_{6}\right]
\end{aligned}
$$

The transformed boundary conditions:

$$
\begin{aligned}
& y_{1}(0)=0, y_{2}(0)=1, y_{3}(0)=\alpha_{1}, y_{4}(0)=1, y_{5}(0)=\alpha_{2}, y_{6}(0)=1 \text { and } y_{7}=\alpha_{3} \text { as } \eta=0 \\
& y_{2}(\infty) \rightarrow \alpha_{4}, \quad y_{4}(\infty) \rightarrow \alpha_{5} \text { and } y_{6}(\infty) \rightarrow \alpha_{6} \text { as } \eta \rightarrow \infty
\end{aligned}
$$

where the unknowns $\alpha_{1}, \alpha_{2}, \alpha_{3}, \alpha_{4}$ and $\alpha_{5}$ have been determined such that

$y_{2}(\infty) \rightarrow \alpha_{4}, \quad y_{4}(\infty) \rightarrow \alpha_{5}$ and $y_{6}(\infty) \rightarrow \alpha_{6}$ as $\eta \rightarrow \infty$

The summary of this technique is that first the boundary value problem has been transfered to an initial value problem. A shooting numerical method has been utilized to presume the values of $\alpha_{1}, \alpha_{2}, \alpha_{3}, \alpha_{4}$ and $\alpha_{5}$ until the boundary conditions are satisfied:

$y_{2}(\infty) \rightarrow \alpha_{4}, \quad y_{4}(\infty) \rightarrow \alpha_{5}$ and $y_{6}(\infty) \rightarrow \alpha_{6}$ as $\eta \rightarrow \infty$ 
By using the ODE45 MATLAB software, the system of equations have been then solved numerically.

\section{Flow Parameters}

The flow parameters are the local skin-friction coefficient $C_{f}$, the local Nusselt number $N u_{x}$ and the local Sherwood number $S h_{x}$, respectively given by:

$C_{f}=\frac{2}{\beta} f_{\eta \eta}(0) R e_{x}^{-\frac{1}{2}}$

$N u_{x}=\frac{1}{2 \beta(1-a t)} \theta_{\eta}(0) R e_{x}^{\frac{3}{2}}$

$S h_{x}=\frac{1}{2 \beta(1-a t)} \phi_{\eta}(0) R e_{x}^{\frac{3}{2}}$

where the local Reynolds number $R e_{x}=\frac{\rho U x}{\mu}$.

\section{Results and Discussions}

The unsteady convection and mass transport over a stretching sheet in a saturated porous medium has been analyzed numerically under the thermal radiation and the chemical reaction effects. The resulting initial value problems involving the set of ODEs (19)-(21) with the boundary conditions (22) - (23) are solved numerically by using the shooting technique with the help of "ODE45 MATLAB" software. The impacts of non-dimensional film thickness $(\gamma)$, unsteadiness parameter $(S)$, Prandlt number $(P r)$, thermal radiation parameter $(R)$ Darcy number $(D a)$, Schmidt number $(S c)$ and chemical reaction parameter $\left(K_{r}\right)$ are displayed in the figures 2 to 14 . To see the impact of non-dimensional thermal radiation parameter, the rest non-dimensional numbers/parameters are considered as constants. The constant values for different parameters/numbers are chosen $\gamma=16, D a=0.6, \operatorname{Pr}=0.71, R=1.0, K_{r}=0.5$, $S c=0.5$, and $S=0.8$.

\section{Velocity distributions for several values of parameters/numbers}

Figure 2 represents the variation of the fluid velocity profile for several values of the Darcy number $(D a)$. The Darcy number is promotional to the kinematic viscosity $(\mu)$. When the Darcy number increases then the kinematic viscosity of the fluid increases. So, the fluid can't move freely. For this reason, the fluid velocity decreases for rising values of the Darcy number. 
This is because that the porous surface produces a resistive type of force for which enhancing the skin-friction coefficient and reduces the fluid velocity.

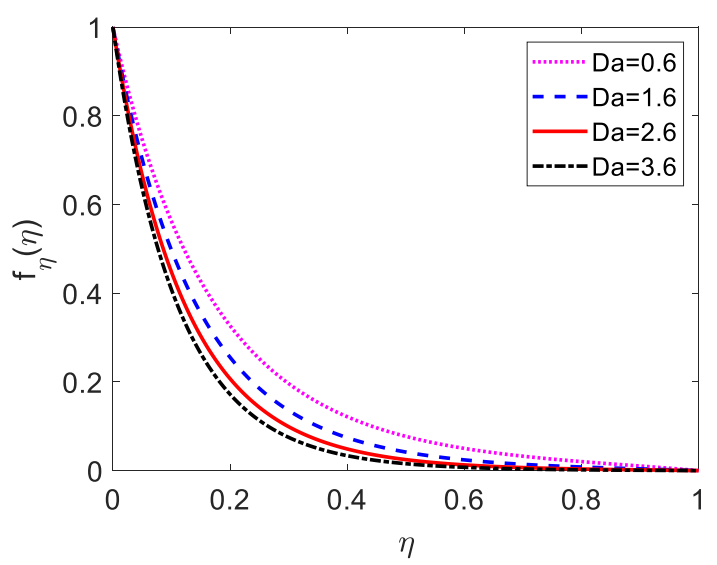

Figure 2: Velocity distribution for Darcy number $(D a)$

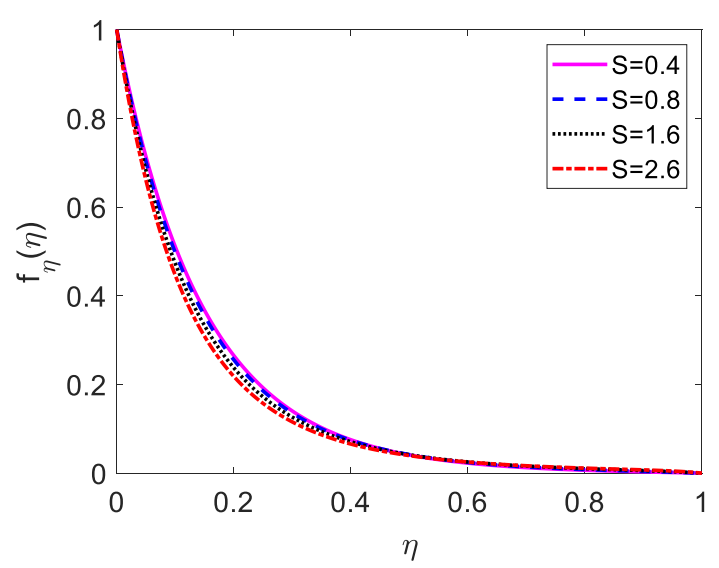

Figure 4: Velocity distribution for unsteadiness parameter $(S)$

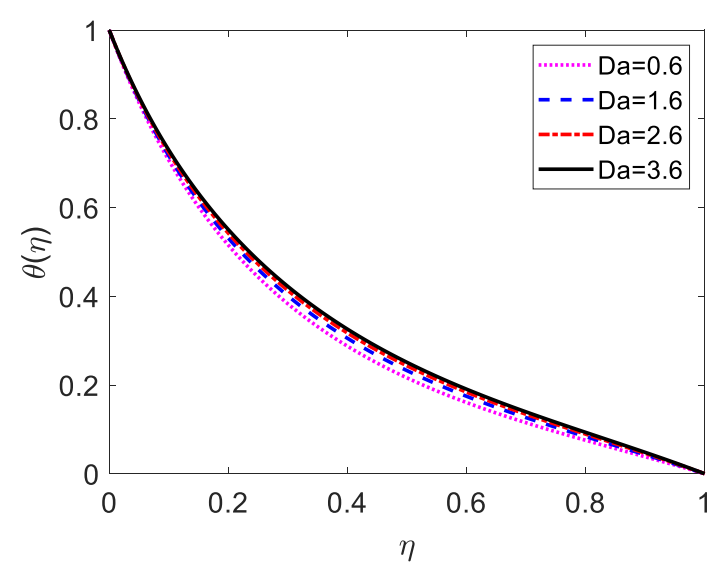

Figure 6: Temperature distribution for Darcy number $(D a)$

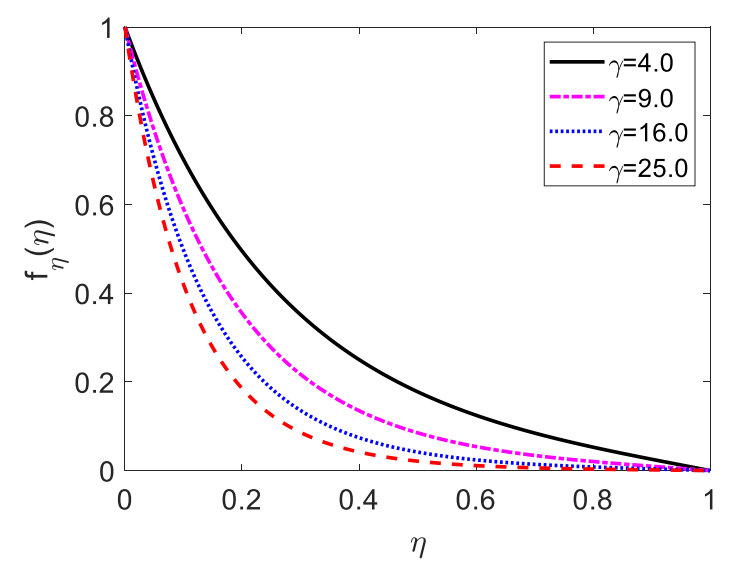

Figure 3: Velocity distribution for dimensionless film thickness $(\gamma)$

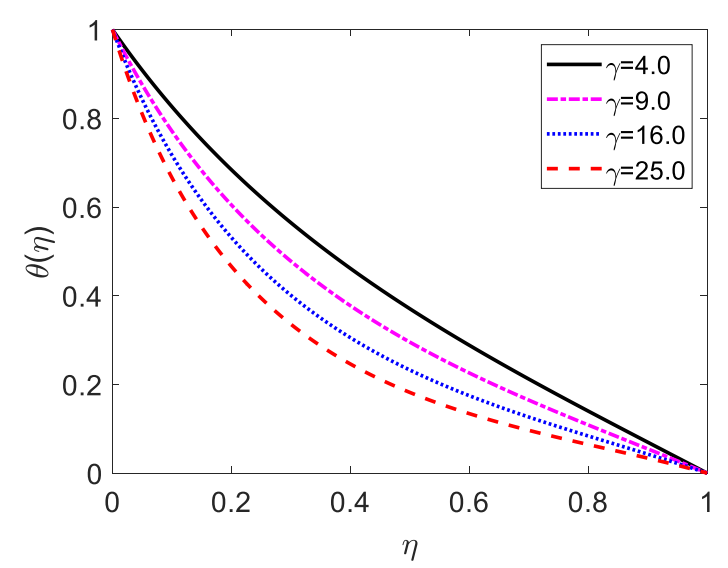

Figure 5: Temperature distribution for dimensionless film thickness $(\gamma)$

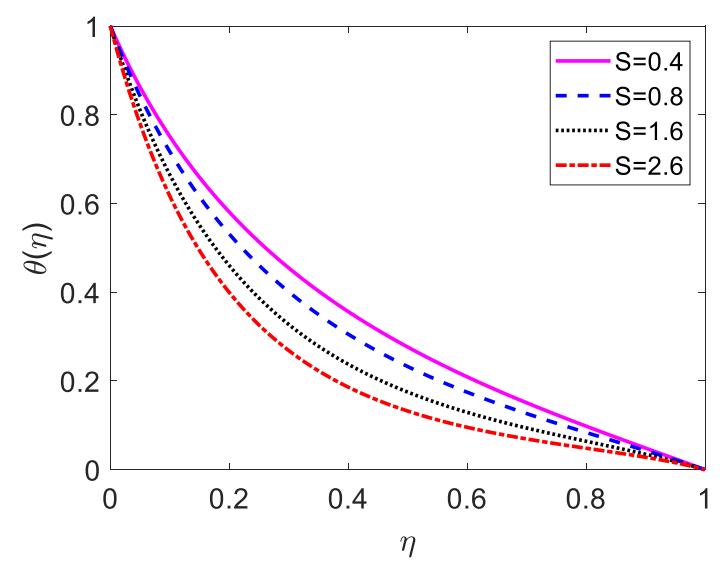

Figure 7: Temperature distribution for unsteadiness parameter $(S)$ 


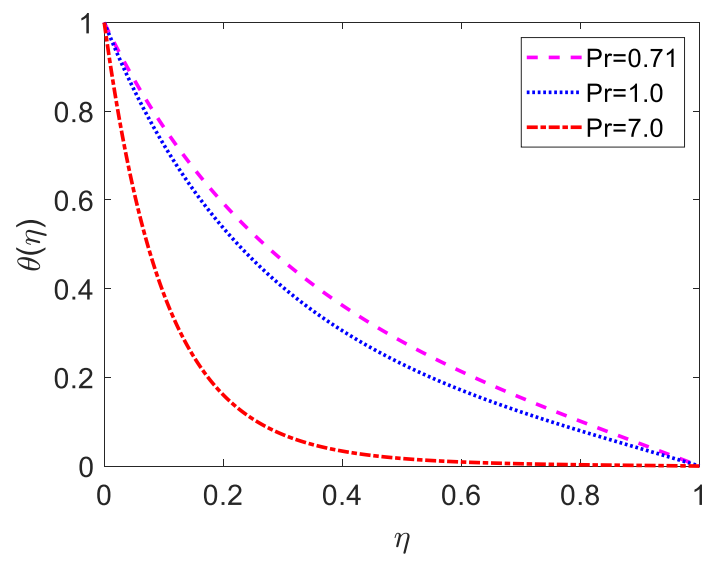

Figure 8: Temperature distribution for Prandtl number $(\mathrm{Pr})$

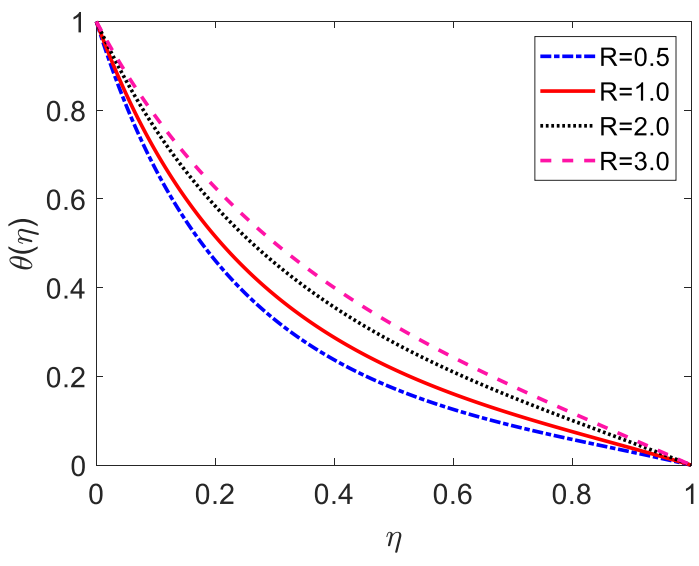

Figure 9: Temperature distribution for thermal radiation parameter $(R)$

Figure 3 represent the impact of dimensionless film thickness $(\gamma)$ on the velocity profile. From

figure. 3 , it is found that the velocity decreases gradually and finally asymptotically leads to zero with the increase of $\eta$ for increasing values of the dimensionless film thickness. The impact of the unsteadiness parameter $(S)$ on the velocity profile is plotted in Figure 4. From figure 4 , it is found that the velocity reduces along the surface for rising values of the unsteadiness parameters.

\section{Temperature distributions for several values of parameters/numbers}

Figure 5 shows the impact of dimensionless film thickness $(\gamma)$ on the temperature distribution. From figure. 5, it is found that for all cases the temperature is starting from 1(one), then temperature decrease gradually and finally asymptotically leads to zero with the increase of $\eta$. Figure 6 represents the variation of the fluid temperature distribution for several values of the Darcy number $(D a)$. Rising values of the Darcy number the fluid temperature reduces. This is due to fact that the Darcy number produces a resistive type of force which causes reduction in the fluid temperature. 


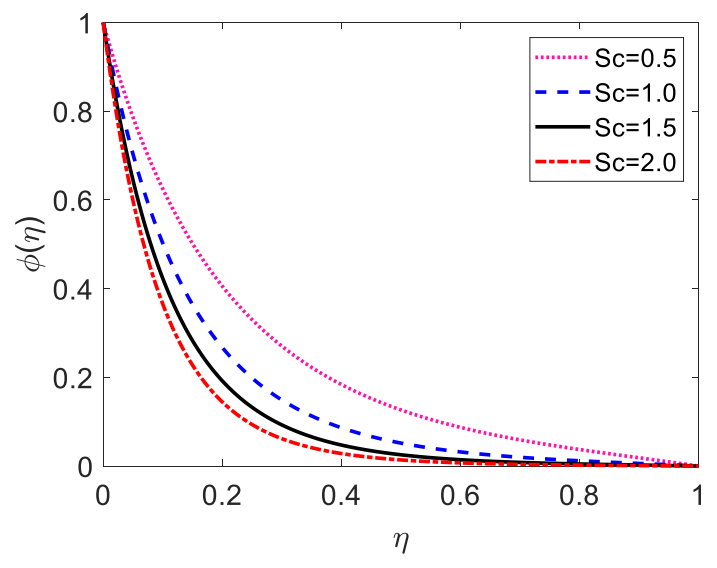

Figure 10: Concentration distribution for Schmidt number $(S c)$

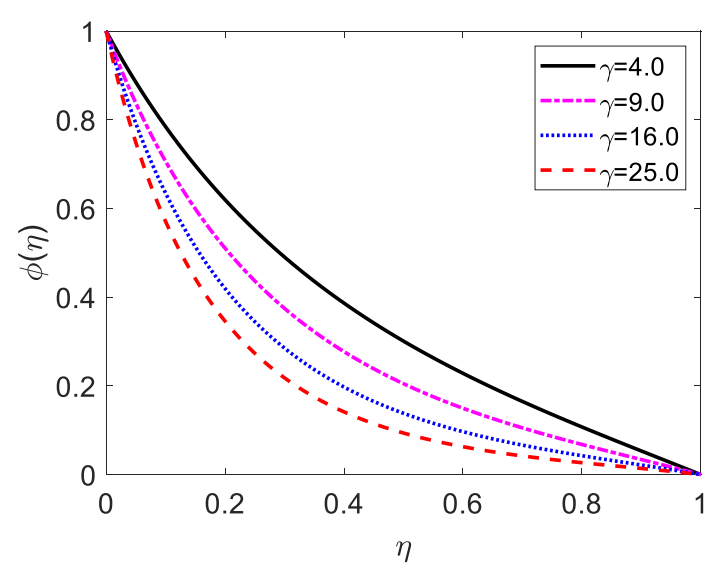

Figure 12: Concentration distribution for dimensionless film thickness $(\gamma)$

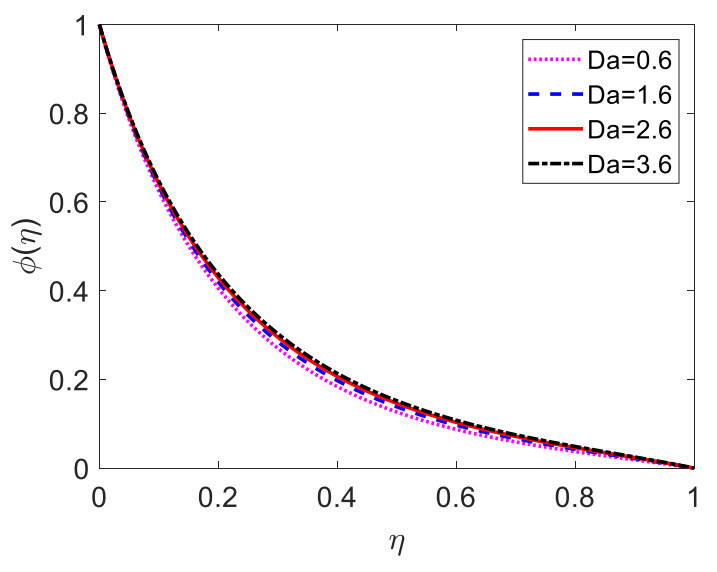

Figure 11: Concentration distribution for Darcy number $(D a)$

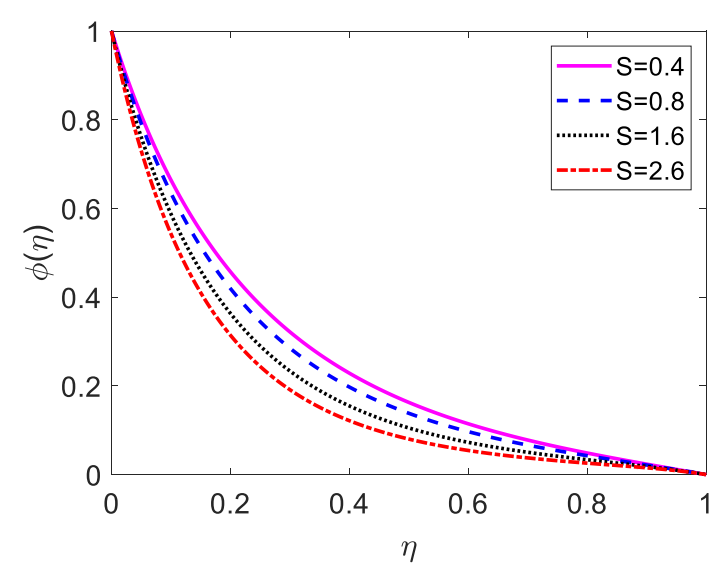

Figure 13: Concentration distribution for unsteadiness parameter $(S)$

Figure 7 illustrates the influence of unsteadiness parameter $(S)$ on the temperature distribution. It is noticed from figure 7 that the velocity enhances along the surface for uplifting values of the unsteadiness parameter. Figure 8 demonstrates the impact of Impact of Prandtl number $(P r)$ on the temperature profile. The Prandtl number $(P r)$ is inversely proportional to the thermal conductivity. The temperature distribution in figure 8 is found to reduce temperature for uplifting values of $\operatorname{Pr}$ (the thermal conductivity decreases). Physically, the lower thermal conductivity has relatively a higher Prandtl number. It reduces heat conduction and so, temperature decreases. Hence the rate of heat transfer enhances for uplifting values of $\operatorname{Pr}$ so that, the temperature profile lessens. The changing of the temperature distribution against $\eta$ for several values of the radiation parameter $\mathrm{R}$ is plotted in Figure 9. It is seen that rising values of the radiation parameter increases the fluid temperature. This is due to fact that enhance in the radiation parameter implies higher surface heat flux and thereby enhancing the temperature of the fluid. 


\section{Impact of several numbers/parameters on concentration distributions}

Figure 10 represents the influence of Schmidt number $(S c)$ on the velocity profile. The molecular (species) diffusivity is inversely proportional to the Schmidt number. When $S c>1$ then the rate of momentum diffusion exceeds the rate of species diffusion. But the opposite behavior for $S c<1$. The concentration (species) and momentum layers will have the same diffusivity rates and thickness for $S c=1$. The concentration distribution in figure 10 is found to reduce the concentration for uplifting values of $S c$. The associated deplete in mass diffusivity outcomes in a small forceful mass transfer which decreases concentration levels and also reduces the thickness of the concentration boundary layer. This is because that mass transfer uses interplay with the species profile and the velocity field in materials can be manipulated via the Schmidt number. The impact of several values of the Darcy number $(D a)$ on the concentration profile is plotted in figure 11. It is revealed that the concentration at any point increase with increase in $D a$. This is due to fact that the permeable surface creates a resistive type of force for which enhancing the concentration of the fluid. Figure 12 represents the effect of dimensionless film thickness $(\gamma)$ on the concentration distribution. It is noticed from figure 12 that uplifting values of the dimensionless film thickness the concentration of the fluid decreases. The changing of the concentration distribution for several values unsteadiness parameter $(S)$ is displayed in figure 13. From figure 13, it is found that the concentration decreases for enhancing values of unsteadiness parameter.

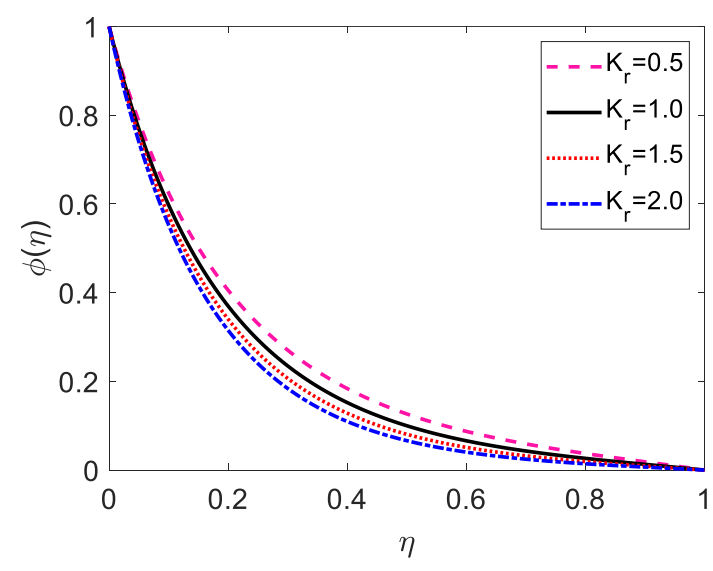

Figure 14: Concentration distribution for chemical reaction parameter $\left(K_{r}\right)$

Figure 14 demonstrates the impact of chemical reaction parameter $\left(K_{r}\right)$ on the concentration distribution. It is noticed from figure 14 that rising values chemical reaction parameter the 
concentration decreases. The impact of chemical reaction parameter is very significant in the concentration field. The interfacial mass transfer rate enhances for rising values of the chemical reaction parameter. Reaction decreases the local concentration, thus enhances its concentration gradient and its flux.

\section{Local skin-friction coefficient, heat and mass transfer rates}

The authors have investigated not only the velocity, temperature, and concentration fields but also the values of the local skin-friction coefficient, heat transfer rate, and mass transfer rate. The authors are given the local skin friction coefficient, heat and mass transfer rates in the following Tables 1 to 7.

Table 1: Impact of Prandtl number $(P r)$ on the local skin-friction coefficient, heat transfer rate and concentration rate when $\gamma=\beta^{2}=16, S=0.8, D a=0.6, R=1.0, S c=0.5$ and $\mathrm{K}_{\mathrm{r}}=$ 0.5

\begin{tabular}{|c|c|c|c|}
\hline$P r$ & $f_{\eta \eta}(0)$ & $-\theta_{\eta}(0)$ & $-\phi_{\eta}(0)$ \\
\hline 0.71 & -5.92429692132274 & 3.67256525641345 & 4.93425485158040 \\
\hline 1.0 & -5.92429692132274 & 4.44956232157320 & 4.93425485158040 \\
\hline 7.0 & -5.92429692132274 & 6.85237323634039 & 4.93425485158040 \\
\hline
\end{tabular}

Tables 1 shows the impact of different values of Prandtl number $(P r)$ on the values of local skin-friction coefficient, heat and mass transfer rates. From the Table 1, it is noticed that the heat transfer rate increases for increasing values of the Prandtl number. The heat transfer rate increases about 22\% and 54\% due to increasing the Prandtl number (0.71-1.0), and (1.0-7.0), respectively. The local skin friction coefficient and the mass transfer rate are remained constants for several values of Prandtl number.

Table 2: Impact of Schmidt number $(S c)$ on the local skin-friction coefficient, heat transfer rate and concentration rate when $\gamma=\beta^{2}=16, S=0.8, D a=0.6, R=1.0, \operatorname{Pr}=$ 0.71 and $\mathrm{K}_{\mathrm{r}}=0.5$

\begin{tabular}{|c|c|c|c|}
\hline Sc & $f_{\eta \eta}(0)$ & $-\theta_{\eta}(0)$ & $-\phi_{\eta}(0)$ \\
\hline 0.5 & -5.92429692132274 & 3.67256525641345 & 4.93425485158040 \\
\hline 1.0 & -5.92429692132274 & 3.67256526551730 & 7.21540963319258 \\
\hline
\end{tabular}




\begin{tabular}{|l|l|l|l|}
\hline 1.5 & -5.92429692132274 & 3.67256526559092 & 8.98750234872303 \\
\hline 2.0 & -5.92429692132274 & 3.67256526560761 & 10.4886272674008 \\
\hline
\end{tabular}

Tables 2 shows the influence of different values of Schmidt number $(S c)$ on the values of local skin-friction coefficient, heat and mass transfer rates. It is found from the Table 2 that the mass transfer rate increases for increasing values of the Schmidt number. The mass transfer rate increases about $46 \%, 25 \%$ and $17 \%$ due to increasing the Schmidt number (0.5-1.0), (1.0-1.5) and (1.5-2.0), respectively. The local skin friction coefficient and the heat transfer rate are remained constants for several values of Schmidt number.

Table 3: Impact of thermal radiation parameter $(R)$ on the local skin-friction coefficient, heat transfer rate and concentration rate when $\gamma=\beta^{2}=16, S=0.8, D a=0.6, S c=0.5, \operatorname{Pr}=$ 0.71 and $\mathrm{K}_{\mathrm{r}}=0.5$

\begin{tabular}{|c|c|c|c|}
\hline$R$ & $f_{\eta \eta}(0)$ & $-\theta_{\eta}(0)$ & $-\phi_{\eta}(0)$ \\
\hline 0.5 & -5.92429692132274 & 4.31205499071904 & 4.93425485158040 \\
\hline 1.0 & -5.92429692132274 & 3.67256525641345 & 4.93425485158040 \\
\hline 1.5 & -5.92429692132274 & 3.24671800461603 & 4.93425485158040 \\
\hline 2.0 & -5.92429692132274 & 2.92554663647185 & 4.93425485158040 \\
\hline
\end{tabular}

Tables 3 shows the impact of different values of thermal radiation parameter $(R)$ on the values of local skin-friction coefficient, heat and mass transfer rates. From the Table 3, it is noticed that the heat transfer rate increases for increasing values of the thermal radiation parameter. The heat transfer rate decreases about $25 \%, 12 \%$ and $10 \%$ due to increasing the thermal radiation parameter $(0.5-1.0),(1.0-1.5)$ and (1.5-2.0), respectively. The local skin friction coefficient and the mass transfer rate are remained constants for several values of thermal radiation parameter.

Table 4: Impact of chemical reaction parameter $\left(K_{r}\right)$ on the local skin-friction coefficient, heat transfer rate and concentration rate when $\gamma=\beta^{2}=16, S=0.8, D a=0.6, S c=0.5$, $\operatorname{Pr}=0.71$ and $R=1.0$

\begin{tabular}{|c|c|c|c|}
\hline$K_{r}$ & $f_{\eta \eta}(0)$ & $-\theta_{\eta}(0)$ & $-\phi_{\eta}(0)$ \\
\hline 0.5 & -5.92429692132274 & 3.67256525641345 & 4.93425485158040 \\
\hline
\end{tabular}




\begin{tabular}{|l|l|l|l|}
\hline 1.0 & -5.92429692132274 & 3.67256525641345 & 5.36455533882288 \\
\hline 1.5 & -5.92429692132274 & 3.67256525641345 & 5.75591368243031 \\
\hline 2.0 & -5.92429692132274 & 3.67256525641345 & 6.11765992656333 \\
\hline
\end{tabular}

Tables 4 shows the influence of several values of chemical reaction parameter $\left(K_{r}\right)$ on the values of local skin-friction coefficient, heat and mass transfer rates. It is found from the Table 4 that the mass transfer rate increases for rising values of the chemical reaction parameter. The mass transfer rate increases about $9 \%, 8 \%$ and $7 \%$ due to increasing the chemical reaction parameter (0.5-1.0), (1.0-1.5) and (1.5-2.0), respectively. The local skin friction coefficient and the heat transfer rate are remained constants for several values of chemical reaction parameter.

Table 5: Impact of Darcy number $(D a)$ on the local skin-friction coefficient, heat transfer rate and concentration rate when $\gamma=\beta^{2}=16, S=0.8, K_{r}=0.5, S c=0.5, \operatorname{Pr}=$ 0.71 and $R=1.0$

\begin{tabular}{|c|c|c|c|}
\hline $\mathrm{Da}$ & $f_{\eta \eta}(0)$ & $-\theta_{\eta}(0)$ & $-\phi_{\eta}(0)$ \\
\hline 0.6 & -5.92429692132274 & 3.67256525641345 & 4.93425485158040 \\
\hline 1.6 & -7.15043776206678 & 3.55937442536495 & 4.82282060611564 \\
\hline 2.6 & -8.19428899449054 & 3.47689953716194 & 4.73935306830790 \\
\hline 3.6 & -9.11905239570234 & 3.41275950963397 & 4.67301091806480 \\
\hline
\end{tabular}

Tables 5 represents the impact of different values of Darcy number $(D a)$ on the values of heat and mass transfer rates and local skin-friction coefficient. It is found from Table 5 that the local skin friction coefficient, heat and mass transfer rates decrease for rising values of the Darcy number. The local skin-friction coefficient decreases about $21 \%, 15 \%$, and $12 \%$ due to increasing the Darcy number (0.6-1.6), (1.6-2.6) and (2.6-3.6), respectively. Rising values of the Darcy number (0.6-1.6), (1.6-2.6) and (2.6-3.6) decrease the heat transfer rate approximately $4 \%, 3 \%$ and $2 \%$ respectively. The mass transfer rate decreases about $4 \%, 3 \%$ and $2 \%$ due to increasing the Darcy number (0.6-1.6), (1.6-2.6) and (2.6-3.6), respectively. 
Table 6: Impact of unsteadiness parameter $(S)$ on the local skin-friction coefficient, heat transfer rate and concentration rate when $\gamma=\beta^{2}=16, D a=0.6, K_{r}=0.5, S c=0.5, \operatorname{Pr}=$ 0.71 and $R=1.0$

\begin{tabular}{|c|c|c|c|}
\hline $\mathrm{S}$ & $f_{\eta \eta}(0)$ & $-\theta_{\eta}(0)$ & $-\phi_{\eta}(0)$ \\
\hline 0.4 & -5.50410947027532 & 3.22894465841629 & 4.48831531702709 \\
\hline 0.8 & -5.92429692132274 & 3.67256525641345 & 4.93425485158040 \\
\hline 1.6 & -6.70068004542515 & 4.41716565429360 & 5.71428846610650 \\
\hline 2.6 & -7.57000503073667 & 5.18858064573773 & 6.55324881773844 \\
\hline
\end{tabular}

Tables 6 represents the impact of different values of unsteadiness parameter $(S)$ on the values of local skin-friction coefficient, heat and mass transfer rates. It is found from Table 6 that the local skin-friction coefficient, heat and mass transfer rates decrease for rising values of the Darcy number. The local skin-friction coefficient reduces about $8 \%, 13 \%$, and $13 \%$ due to increasing the unsteadiness parameter $(0.4-0.8),(0.8-1.6)$ and (1.6-2.6), respectively. Rising values of the unsteadiness parameter (0.4-0.8), (0.8-1.6) and (1.6-2.6) increase the heat transfer rate approximately $14 \%, 21 \%$ and $18 \%$ respectively. The mass transfer rate increases about $10 \%, 16 \%$ and $15 \%$ due to increasing the unsteadiness parameter $(0.4-0.8),(0.8-1.6)$ and (1.62.6), respectively.

Table 7: Impact of dimensionless film thickness $(\gamma)$ on the local skin-friction coefficient, heat transfer rate and concentration rate when $S=0.8, D a=0.6, K_{r}=0.5, S c=0.5, \operatorname{Pr}=$ 0.71 and $R=1.0$

\begin{tabular}{|c|c|c|c|}
\hline$\gamma$ & $f_{\eta \eta}(0)$ & $-\theta_{\eta}(0)$ & $-\phi_{\eta}(0)$ \\
\hline 4.0 & -2.98896116936767 & 1.98068297647479 & 2.52816963816624 \\
\hline 9.0 & -4.44667896305692 & 2.79869440173499 & 3.71311183204355 \\
\hline 16.0 & -5.92429692132274 & 3.67256525641345 & 4.93425485158040 \\
\hline 25.0 & -7.40481834028061 & 4.56988107599930 & 6.16432202296863 \\
\hline
\end{tabular}

Tables 7 represents the impact of different values of dimensionless film thickness $(\gamma)$ on the values of local skin-friction coefficient, heat and mass transfer rates. It is found from Table 7 that the local skin-friction coefficient, heat and mass transfer rates decrease for rising values of 
the dimensionless film thickness. The local skin-friction coefficient reduces about $49 \%, 34 \%$, and $25 \%$ due to increasing the dimensionless film thickness (4.0-9.0), (9.0-16.0) and (16.025.0), respectively. Rising values of the dimensionless film thickness (4.0-9.0), (9.0-16.0) and (16.0-25.0) increase the heat transfer rate approximately $43 \%, 33 \%$ and $25 \%$ respectively. The mass transfer rate increases about $47 \%, 33 \%$ and $25 \%$ due to increasing the dimensionless film thickness (4.0-9.0), (9.0-16.0) and (16.0-25.0), respectively.

\section{Comparison}

The present research results have been compared with Khader and Megahed [21]. The Comparison of the local skin-friction coefficient and the heat transfer rate has been shown in Table 8 and Table 9, respectively. From these tables, it is found that the comparisons of the present numerical outcomes show a good agreement with previously published outcomes under the special cases. These comparisons ensure the accuracy and validity of the present research work.

Table 8: Comparison for values of $f_{\eta \eta}(0)$ and $\theta_{\eta}(0)$ for different values of $D a$ and $\gamma$ when $C=0$

\begin{tabular}{|c|c|c|c|c|c|}
\hline$D a$ & $\gamma$ & $\begin{array}{c}f_{\eta \eta}(0) \\
\text { Khader and Megahed [21] }\end{array}$ & $\begin{array}{c}f_{\eta \eta}(0) \\
\text { Present work }\end{array}$ & $\begin{array}{c}-\theta_{\eta}(0) \\
\text { Khader and Megahed [21] }\end{array}$ & $\begin{array}{r}-\theta_{\eta}(0) \\
\text { Present work }\end{array}$ \\
\hline 0.0 & 5.56880 & -2.91668 & -3.00844 & 2.61712 & 2.63459 \\
\hline 0.2 & 4.91978 & -2.90572 & -2.99903 & 2.42829 & 2.46745 \\
\hline 0.4 & 4.43018 & -2.90428 & -2.99925 & 2.27474 & 2.31605 \\
\hline 0.6 & 4.05661 & -2.90234 & -3.00912 & 2.14948 & 2.19209 \\
\hline
\end{tabular}

Table 9: Comparison for values of $f_{\eta \eta}(0)$ and $\theta_{\eta}(0)$ for different values of $R$ and $\gamma$ when $C=0$

\begin{tabular}{|c|c|c|c|c|c|}
\hline$R$ & $\gamma$ & $\begin{array}{c}f_{\eta \eta}(0) \\
\text { Khader and Megahed [21] }\end{array}$ & $\begin{array}{c}f_{\eta \eta}(0) \\
\text { Present work }\end{array}$ & $\begin{array}{c}-\theta_{\eta}(0) \\
\text { Khader and Megahed [21] }\end{array}$ & $\begin{array}{c}-\theta_{\eta}(0) \\
\text { Present work }\end{array}$ \\
\hline 1.0 & 4.919789 & -2.9057 & -2.99903 & 2.42829 & 2.46745 \\
\hline 3.0 & 4.919789 & -2.9057 & -2.99903 & 1.54551 & 1.56421 \\
\hline 5.0 & 4.919789 & -2.9057 & -2.99903 & 1.15024 & 1.16780 \\
\hline 7.0 & 4.919789 & -2.9057 & -2.99903 & 0.91955 & 1.05745 \\
\hline
\end{tabular}




\section{Conclusions}

The unsteady convection and mass transport over a stretching sheet in a saturated porous medium has been analyzed numerically under the thermal radiation and the chemical reaction effects. From the above numerical simulations, the below conclusions can be illustrated:

- The local skin friction coefficient decreases about $21 \%, 8 \%$, and $34 \%$ due to increasing, Darcy number (0.6-1.6), unsteadiness parameter (0.4-0.8) and dimensionless film thickness (9.0-16.0), respectively.

- Uplifting values of the Prandtl number (0.71-1.0) and dimensionless film thickness (9.0-16.0) enhance the heat transfer rate by $22 \%$ and $33 \%$, respectively but decrease about $25 \%, 4 \%$, and $14 \%$ for enhancing thermal radiation parameter (0.5-1.0), Darcy number (0.6-1.6), and unsteadiness parameter (0.4-0.8), respectively.

- The mass transfer rate enhances about $9 \%, 14 \%$, and $33 \%$ due to enhancing the chemical reaction parameter (0.5-1.0), the unsteadiness parameter (0.4-0.8), and dimensionless film thickness (9.0-16.0), respectively. Besides, increasing Schmidt number (0.5-1.0) and Darcy number (0.6-1.6) decreases the mass transfer rate by $46 \%$ and $4 \%$, respectively.

The result of this paper can be helpful for chemical processing equipment and tinning of copper wires, polymer processing, pollution of ground water, geophysical thermal insulation, reactor fluidization, welding, geothermal extraction, crystal growing, etc.

\section{Data Availability}

Data can be accessible upon request to corresponding author (hasanuzzaman@math.kuet.ac.bd).

\section{References}

1. Wang, C. Y. (1990). Liquid film on an unsteady stretching surface. Quarterly of applied Mathematics, 48(4), 601-610.

2. Dandapat, B. S., Santra, B., \& Andersson, H. I. (2003). Thermocapillarity in a liquid film on an unsteady stretching surface. International Journal of Heat and Mass Transfer, 46(16), 3009-3015.

3. Cheng, P. (1977). Combined free and forced convection flow about inclined surfaces in porous media. Int. J. Heat Mass Transfer, 20, 807-814. 
4. Merkin, J. H. (1986). On dual solutions occurring in mixed convection in a porous medium. Journal of engineering Mathematics, 20(2), 171-179.

5. Wright, S. D., Ingham, D. B., \& Pop, I. (1996). On natural convection from a vertical plate with a prescribed surface heat flux in porous media. Transport in porous media, 22(2), 181-193.

6. Yih, K. A. (1997). The effect of uniform lateral mass flux on free convection about a vertical cone embedded in a saturated porous medium. International communications in heat and mass transfer, 24(8), 1195-1205.

7. Gorla, R. S. R., Slaouti, A., \& Takhar, H. S. (1997). Mixed convection in nonNewtonian fluids along a vertical plate in porous media with surface mass transfer. International Journal of Numerical Methods for Heat \& Fluid Flow.

8. Crane, L. J. (1970). Flow past a stretching plate. Zeitschrift für angewandte Mathematik und Physik ZAMP, 21(4), 645-647.

9. Gupta, P. S., \& Gupta, A. S. (1977). Heat and mass transfer on a stretching sheet with suction or blowing. The Canadian journal of chemical engineering, 55(6), 744-746.

10. Andersson, H. I., Aarseth, J. B., \& Dandapat, B. S. (2000). Heat transfer in a liquid film on an unsteady stretching surface. International Journal of Heat and Mass Transfer, 43(1), 69-74.

11. Abel, M. S., Mahesha, N., \& Tawade, J. (2009). Heat transfer in a liquid film over an unsteady stretching surface with viscous dissipation in presence of external magnetic field. Applied Mathematical Modelling, 33(8), 3430-3441.

12. Noor, N. F. M., \& Hashim, I. (2010). Thermocapillarity and magnetic field effects in a thin liquid film on an unsteady stretching surface. International Journal of Heat and Mass Transfer, 53(9-10), 2044-2051.

13 Song, Y. Q., Khan, M. I., Qayyum, S., Gowda, R. P., Kumar, R. N., Prasannakumara, B. C., ... \& Chu, Y. M. (2021). Physical impact of thermo-diffusion and diffusionthermo on Marangoni convective flow of hybrid nanofluid (MnZiFe2O4-NiZnFe2O4$\mathrm{H} 2 \mathrm{O}$ ) with nonlinear heat source/sink and radiative heat flux. Modern Physics Letters $B, 35(22), 2141006$.

14. Faisal, M. T. R., \& Islam, A. K. M. S. (2006). Primitive variable approach to calculate separation point in laminar boundary layer along a flat plate. ARPN Journal of Engineering and Applied Science, 1(3). 
15. Sweilam, N. H., Khader, M. M., \& Nagy, A. M. (2011). Numerical solution of twosided space-fractional wave equation using finite difference method. Journal of Computational and Applied Mathematics, 235(8), 2832-2841.

16. Cheng, W. T., \& Lin, C. H. (2008). Unsteady mass transfer in mixed convective heat flow from a vertical plate embedded in a liquid-saturated porous medium with melting effect. International Communications in Heat and Mass Transfer, 35(10), 1350-1354.

17. Johnston, H., \& Liu, J. G. (2002). Finite difference schemes for incompressible flow based on local pressure boundary conditions. Journal of Computational Physics, 180(1), 120-154.

18. Chamkha, A.J., Ahmed, S.A., Aloraier, A.S., 2010. Melting and radiation effects on mixed convection from a vertical surface embedded in a non-Newtonian fluid saturated non-Darcy porous medium for aiding and opposing eternal flows. Int. J. Phys. Sci. 5, $1212-1224$.

19 Hasanuzzaman, M., Kabir, M. A., \& Ahmed, M. T. (2021). Transpiration effect on unsteady natural convection boundary layer flow around a vertical slender body. Results in Engineering, 12, 100293.

20 Hasanuzzaman, M., Azad, M., Kalam, A., \& Hossain, M. (2021). Effects of Dufour and thermal diffusion on unsteady MHD free convection and mass transfer flow through an infinite vertical permeable sheet. SN Applied Sciences, 3(12), 1-11.

21. Khader, M. M., \& Megahed, A. M. (2013). Numerical simulation using the finite difference method for the flow and heat transfer in a thin liquid film over an unsteady stretching sheet in a saturated porous medium in the presence of thermal radiation. Journal of King Saud University-Engineering Sciences, 25(1), 29-34.

22. Raptis, A. (1998). Flow of a micropolar fluid past a continuously moving plate by the presence of radiation. International Journal of Heat and Mass Transfer, 41(18), 28652866.

23. Raptis, A. (1999). Radiation and viscoelastic flow. International Communications in Heat and Mass Transfer, 26(6), 889-895. 